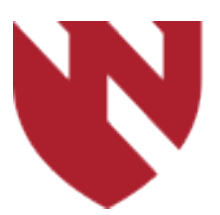

\title{
Diagnostic Quality and Radiation Dose for Pulmonary Embolism Protocol Computed Tomography: ASIR-V vs. Standard Filtered Back Projection
}

\author{
Lei Yu \\ University of Nebraska Medical Center \\ Antonia Gurney \\ University of Nebraska Medical Center \\ Kelly Dea \\ University of Nebraska Medical Center \\ Neil Hansen \\ University of Nebraska Medical Center \\ Valerie Shostrom \\ University of Nebraska Medical Center
}

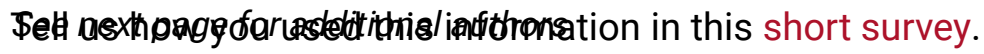

Follow this and additional works at: https://digitalcommons.unmc.edu/gmerj

Part of the Higher Education Commons, and the Medicine and Health Sciences Commons

\section{Recommended Citation}

Yu, L., Gurney, A., Dea, K., Hansen, N., Shostrom, V., Are, C., , Zajicek, A. Diagnostic Quality and Radiation Dose for Pulmonary Embolism Protocol Computed Tomography: ASIR-V vs. Standard Filtered Back Projection. Graduate Medical Education Research Journal. 2020 Sep 29; 2(1).

https://digitalcommons.unmc.edu/gmerj/vol2/iss1/30

This Conference Proceeding is brought to you for free and open access by DigitalCommons@UNMC. It has been accepted for inclusion in Graduate Medical Education Research Journal by an authorized editor of DigitalCommons@UNMC.For more information, please contact digitalcommons@unmc.edu. 


\section{Diagnostic Quality and Radiation Dose for Pulmonary Embolism Protocol Computed Tomography: ASIR-V vs. Standard Filtered Back Projection}

\section{Creative Commons License}

\section{c) (1)@ $\Theta$}

This work is licensed under a Creative Commons Attribution-Noncommercial-No Derivative Works 4.0 License.

Authors

Lei Yu, Antonia Gurney, Kelly Dea, Neil Hansen, Valerie Shostrom, Chandrakanth Are, and Anna Zajicek 


\section{Sleep Improvement Program and Academic Performance}

Matthew Tingle ${ }^{1}$, Liz Wellsandt ${ }^{2}$, Kari Nelson ${ }^{3}$, RJ Barber ${ }^{1}$, Beau Konigsberg ${ }^{1}$, Maxwell Lydiatte ${ }^{4}$, Cale Ewald ${ }^{4}$, Matthew McManigal ${ }^{2}$, Matthew Tao ${ }^{1}$

1 University of Nebraska Medical Center, Department of Orthopedic Surgery

${ }^{2}$ University of Nebraska Medical Center, College of Allied Health Professions, Physical Therapy

${ }^{3}$ University of Nebraska Medical Center, Office of Graduate Medical Education

${ }^{4}$ University of Nebraska Medical Center, College of Medicine

\section{Mentor: Matthew Tao}

Program: Orthopedic Surgery

Type: Original Research

Background: The aim of this study is to investigate the impact of improved sleep quality and extension can have on academic performance in medical students. Additional outcome measures include evaluating mood (Profile of Mood States) and sleepiness (Epworth Sleepiness Scale). Our hypothesis is that improved sleep quality and extension will significantly improve test scores, mood and daytime sleepiness.

Methods: This is a pilot study involving 20 first-year medical students. They will be randomized into either control or intervention groups. Both groups will be monitored for baseline sleep characteristics. The intervention group will then undergo a training program aimed to improve sleep quality and extension. Subjects will be monitored via actigraphy devices (Actigraph wGT3x-BT) on the dominant hand at all times as well as daily, subjective sleep logs. The primary outcome will be raw test scores at the typical testing intervals based on the curriculum. ESS questionnaire will be administered at the beginning and end of the study, and the POMS will be administered weekly.

Results and Conclusion: Pending https://doi.org/10.32873/unmc.dc.gmerj.2.1.027

\section{The Clinical Relevance of Cement Volume in Percutaneous Vertebral Augmentation} Ravi G. Mirpuri', Michael J. Warden'1, Virginia Hardie', Madhuri Are ${ }^{1}$

${ }^{1}$ University of Nebraska Medical Center, Department of Anesthesiology

\section{Mentor: Madhuri Are}

Program: Anesthesiology

Type: Original Research

Background: Vertebroplasty and Kyphoplasty are two forms of percutaneous vertebral augmentation (PVA), in which polymethylmethacrylate cement is used to stabilize vertebral compression fractures (VCF). This study sought to evaluate the relationship between cement volume and clinical outcomes, including pain reduction, opioid use, and complication rate.

Methods: Retrospective chart review produced 88 patients who received PVA at a tertiary care outpatient pain clinic. Cement volume, type of PVA, gender, level (thoracic vs lumbar) were collected, as well as clinical outcomes of numeric pain score (NPS) reduction, opioid percent change (OPC), and complications. Both pre-procedure and postprocedure (between 2-4 weeks) data were collected.

Results: Sixty-four patients (72.7\%) had statistically significant NPS reduction of $\geq 50 \%$ pain $(\mathrm{p}<.001)$. The mean NPS reduction was $4.45(\mathrm{p}<.0001)$. Also sixtythree out of seventy-two patients who previously used opioids demonstrated some form of opioid reduction which was significant $(\mathrm{p}<.0001)$. The mean opioid reduction measured across all baseline opioid users was $48 \%$. No statistically significant differences in NPS or OPC were seen across gender or level type. Linear regression using pre NPS as a covariate showed cement volume did not have a significant effect on NPS change or opioid reduction $(\mathrm{p}=0.173$ and $\mathrm{p}=.299$ respectively). Cement leakage occurred as a complication in $10.2 \%$ of the patients, although only one complication was symptomatic.

Conclusion: Cement volume does not correlate with clinical outcomes of NPS reduction or OPC. Furthermore, our study reinforced PVA as a beneficial treatment for reducing pain and opioid consumption in patients with painful VCF.

https://doi.org/10.32873/unmc.dc.gmerj.2.1.028

\section{Diagnostic Quality and Radiation Dose for Pulmonary Embolism Protocol Computed Tomography: ASIR-V vs. Standard Filtered Back Projection \\ Lei Yu ${ }^{1 *}$, Antonia Gurney ${ }^{1 *}$, Kelly Dea ${ }^{1 *}$, Neil Hansen ${ }^{1}$, Valerie Shostrom², Chandrakanth Are ${ }^{3}$, Anna Zajicek $^{1}$ \\ ${ }^{1}$ University of Nebraska Medical Center, Department of Radiology \\ ${ }^{2}$ University of Nebraska Medical Center, College of Public Health, Department of Biostatistics \\ ${ }^{3}$ University of Nebraska Medical Center, Office of Graduate Medical Education \\ *authors contributed equally to the work}

Mentor: Anna Zajicek

Program: Radiology

Type: Review/Meta-analysis

Background: Pulmonary embolism (PE) is the third leading cause of acute cardiovascular disease, contributes to 250,000 American hospitalizations yearly. CT pulmonary angiography (CTPA) is the first-line imaging choice for suspected PE, however radiation exposure remains a concern. We compared the Dose-Length Product (DLP) in CTPA performed using adaptive statistical iterative reconstruction - V (ASIR-V) versus older filtered back projection (FBP) technique, while also evaluating the ability of ASIR-V to maintain diagnostic accuracy.

Methods: A retrospective review was performed of all CTPA scans completed for suspected PE during a one-year period at our institution. A total of 1095 studies met criteria for inclusion. Five-hundred and thirty-three $(48.7 \%)$ of these were performed on a CT scanner utilizing ASIR-V (GE Healthcare), and $562(51.3 \%)$ were performed on an older CT scanner utilizing standard FBP (Lightspeed VCT, GE Healthcare). Doselength product (DLP) and demographic data were recorded. Original radiology reports from board-certified radiologists for each exam were reviewed. Diagnostic quality was determined by usage of specified 
caveat phrases in the original radiology interpretation. The studies were categorized into diagnostic, technically limited, or nondiagnostic studies.

Results: Studies performed using ASIR-V (375.6 $\left.\mathrm{mGy}^{*} \mathrm{~cm}[255.9,501.4]\right)$ had a significantly lower DLP compared to those using FBP (695.3 mGy*cm [410.6, 1110.0]). The diagnostic quality was superior when performed using ASIR-V, with 90\% (481/533) of studies being diagnostic, compared to $80 \%$ $(452 / 562 ; \mathrm{P}<.0001)$ in the FBP group.

Conclusions: CTPA performed using ASIR-V both reduced radiation dose as well as improved diagnostic confidence compared to CTPA performed using FBP.

https://doi.org/10.32873/unmc.dc.gmerj.2.1.029

\section{References}

1 Moore, Alastair JE, et al. "Imaging of acute pulmonary embolism: an update." Cardiovascular Diagnosis and Therapy 8.3 (2018): 225.

2 Roshkovan, Leonid, and Harold Litt. "State-of-the-art imaging for the evaluation of pulmonary embolism." Current Treatment Options in Cardiovascular Medicine. 20.9 (2018): 71

3 Lenga, Lukas, et al. "Single-and dual-energy CT pulmonary angiography using second-and thirdgeneration dual-source CT systems: comparison of radiation dose and image quality." European Radiology 29.9 (2019): 4603-4612.

4 Singh, Sarabjeet, et al. "Adaptive statistical iterative reconstruction technique for radiation dose reduction in chest CT: a pilot study." Radiology. 259.2 (2011): 565-573.

5 Kwon, Heejin, et al. "The adaptive statistical iterative reconstruction-V technique for radiation dose reduction in abdominal CT: comparison with the adaptive statistical iterative reconstruction technique." The British Journal of Radiology. 88.1054 (2015): 20150463.
6 Qiu, D., and E. Seeram. "Does iterative reconstruction improve image qual-ity and reduce dose in computed tomography." Radiol Open J. 1.2 (2016): 42-54.Desai

7 Gaurav S., et al. "Impact of iterative reconstruction on image quality and radiation dose in multidetector CT of large body size adults." European Radiology. 22.8 (2012): 1631-1640.

\section{Expanded Mutation Profiling in Appendix Peritoneal Metastasis Has Prognostic and Therapeutic Utility When Managed with Cytoreductive Surgery/Hyperthermic Intraperitoneal Chemotherapy (CRS/HIPEC)} Chunmeng Zhang ${ }^{1}$, Ben Plambeck², Maggie Moore ${ }^{2}$, Alexander Tu², Ryan Miku², Valerie Shostrom ${ }^{3}$, Krista Brown ${ }^{4}$, Allison Cushman-Volkoun ${ }^{5}$, Benjamin Swanson ${ }^{5}$, Jason Foster ${ }^{1}$

${ }^{1}$ University of Nebraska Medical Center, Department of Surgery

2University of Nebraska Medical Center, College of Medicine

${ }^{3}$ University of Nebraska Medical Center, College of Public Health, Department of Biostatistics

${ }^{4}$ University of Nebraska Medical Center, College of Public Health, Department of Epidemiology

${ }^{5}$ University of Nebraska Medical Center, Department of Pathology

Mentor: Jason Foster

Program: General Surgery

Type: Original Research

Background: The identification of relevant biological pathways and mutations is integral to improving outcomes in appendix peritoneal metastases (APM). Interrogation of cancers with Next Generation Sequencing (NGS) 50-gene mutation panels has become more widely utilized identifying prognostic and actionable mutations. This study is a dedicated analysis of the value of expanded mutation analysis in APM.

Methods: The IRB approved study included 51 APM patients where data was retrospectively collected from a CRS/HIPEC registry treated 2012-2018. Standard clinical 50 -gene NGS analysis was performed in CLIA approved lab. All patients underwent CRS/ HIPEC with mitomycin $\mathrm{C}$ delivered for 90 minutes at 41-42 C. Peritoneal Cancer Index (PCI), Completeness of Cytoreduction (CC) score, length of stay, progression free survival (PFS), overall survival (OS) were collected along with the rates and types of mutation in APM. OS and PFS analyses were performed on all, high grade (HG), and low grade (LG) APM, specifically evaluating the impact of smad4 and p53 mutations on survival.
Results: Eighty-four percent of APM had a mutation identified with $58 \%$ of cases harboring $\geq 2$ mutations. Kras was most frequent, $66 \%$ of APM (88\% LG 44\% HG) and GNAS identified in $88 \%$ of LG APM. Smad 4 or p53 mutation occurred in $25 \%$ of APM and a significant reduction in OS in all APM ( 22 vs 88 months $\mathrm{p}=0.0026)$ and HG APM (20 vs 47 months $\mathrm{p}=0.0502$ ) was observed. Smad4 mutation was also associated with a significant reduction in PFS APM ( $p=0.0192)$. Actionable mutations were identified in $70 \%$ of APM.
Conclusion: Smad4 and p53 mutations were associated with more aggressive APM and maybe a useful tool in patient selection and outcome. Expanded mutation profiles is valuable in APM and further application is warranted. Research in Kras, p53 and smad4 pathways and drug development will benefit APM.

https://doi.org/10.32873/unmc.dc.gmerj.2.1.030

\section{Table 1.}

p53 and/or SMAD4 mutations are associated with significantly reduced overall survival (OS) in all patients and high grade (HG) subgroup; SMAD4 mutations alone was associated with significantly shortened progression free survival (PFS) in all patients and high-grade subgroup. LG, low grade.

\begin{tabular}{lccc} 
& $\begin{array}{c}\text { WT P53 \& SMAD4 } \\
\text { (months) }\end{array}$ & $\begin{array}{c}\text { +P53 OR +SMAD4 } \\
\text { (months) }\end{array}$ & P VALUE \\
\hline OS $_{\mathrm{ALL}}$ & 88 & 22 & $<0.01$ \\
\hline OS $_{\mathrm{HG}}$ & 47 & 20 & 0.05 \\
\hline & $\begin{array}{c}\text { WT SMAD4 } \\
\text { (months) }\end{array}$ & 10 & $\begin{array}{c}\text { +SMAD4 } \\
\text { (months) }\end{array}$ \\
\hline PFS $_{\mathrm{ALL}}$ & 6.5 & 3.5 & 0.02 \\
\hline PFS $_{\mathrm{HG}}$ & 20 & 1.5 & 0.01 \\
\hline $\mathrm{PFS}_{\mathrm{LG}}$ & & 7.5 & 0.09
\end{tabular}

